where $s_{v}^{*}\left(\theta_{0}\right), v=0,1, \cdots, n$, is the sequence $\left|s_{v}\left(\theta_{0}\right)-s\right|$ in decreasing order.

\title{
REFERENCES
}

1. G. H. Hardy and I. E. Littlewood, Some new convergence criteria for Fourier series, Annali, Scuola Normale Superiore, Pisa, (2), vol. 3 (1934), pp. 43-62.

2. - Notes on the theory of series (XVIII): On the convergence of Fourier series, Proceedings of the Cambridge Philosophical Society, vol. 31 (1935), pp. 317323.

3. A. Zygmund, Trigonometrical Series, 1935.

University of CincinNati

\section{THE BASIC ANALOGUE OF KUMMER'S THEOREM ${ }^{1}$}

\section{J. A. DAUM}

1. Introduction. About one hundred years ago, E. E. Kummer ${ }^{2}$ proved the formula

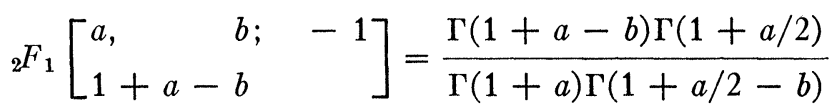

which has since been known as Kummer's theorem. This appears to be the simplest relation involving a hypergeometric function with argument $(-1)$.

All the relations in the theory of hypergeometric series ${ }_{r} F_{s}$ which have analogues in the theory of basic series ${ }^{3}$ are those in which the argument is $(+1)$. Apparently, there has been no successful attempt to establish the basic analogue of any formula involving a function ${ }_{r} F_{s}(-1)$. Since Kummer's theorem is fundamental in the proofs of numerous relations between hypergeometric functions of argument $(-1)$, it seemed desirable that an attempt be made to prove the basic analogue of Kummer's theorem and to investigate the possibility of obtaining new relations in basic series with arguments corresponding to the argument $(-1)$ in the classical case.

In this paper, the basic analogue of Kummer's theorem is obtained

Received by the editors November 27, 1941.

${ }^{1}$ The results presented in this paper are included in a dissertation for the doctorate, University of Nebraska, 1941.

${ }^{2}$ E. E. Kummer, Ueber die hypergeometrische Reihe, Journal für die reine und angewandte Mathematik, vol. 15 (1836), pp. 39-83.

${ }^{3}$ W. N. Bailey, Generalized Hypergeometric Series, Cambridge Tract, no. 32, chap. 8. 
by a simple specialization of a result due to Bailey. ${ }^{4}$ The existence of a basic analogue of Dixon's theorem ${ }^{5}$ is discussed and a new type of relation connecting a modified basic series and one of the conventional type is presented.

2. Derivation of the basic analogue of Kummer's theorem. In the formula :4

${ }_{6} \Phi_{5}\left[\begin{array}{c}a, q a^{1 / 2},-q a^{1 / 2}, d, \quad e, \quad f ; a q / d e f \\ a^{1 / 2},-a^{1 / 2}, a q / d, a q / e, a q / f\end{array}\right]$

$$
=\frac{\Omega(a q / d) \Omega(a q / e) \Omega(a q / f) \Omega(a q / d e f)}{\Omega(a q) \Omega(a q / e f) \Omega(a q / d f) \Omega(a q / d e)}
$$

where ${ }^{6}$

$$
\Omega(z)=\prod_{n=0}^{\infty} \frac{1-q^{n+1}}{1-z q^{n}},
$$

put $e=a^{1 / 2}, f=-a^{1 / 2}$, and $d=b$, and obtain

$$
{ }_{2} \Phi_{1}\left[\begin{array}{cc}
a, b ; & -q / b \\
a q / b
\end{array}\right]=\frac{\Omega(a q / b) \Omega\left(q a^{1 / 2}\right) \Omega\left(-q a^{1 / 2}\right) \Omega(-q / b)}{\Omega(a q) \Omega(-q) \Omega\left(q a^{1 / 2} / b\right) \Omega\left(-q a^{1 / 2} / b\right)} .
$$

This is the basic analogue of Kummer's theorem. It should be noted that it cannot be obtained from the basic analogue of Gauss's theorem. ${ }^{7}$

3. Application. The basic analogue of Kummer's theorem immediately suggests the existence of the basic analogue of Dixon's theorem. It has been shown by Watson ${ }^{8}$ that Dixon's theorem, which sums a general well poised ${ }_{3} F_{2}$ with unit argument, can be derived from the theorems of Gauss and Kummer by means of an interchange in the order of summation.

Unfortunately, this method, when applied to the basic analogues, does not lead to an analogue of Dixon's theorem. The difficulty is due to an essential difference in the properties of the omega and

${ }^{4}$ W. N. Bailey, Series of hypergeometric type which are infinite in both directions, Quarterly Journal of Mathematics, Oxford, vol. 7 (1936), p. 105.

${ }^{5}$ A. G. Dixon, Summation of certain series, Proceedings of the London Mathematical Society, (1), vol. 35 (1903), pp. 285-289.

${ }^{6}$ N. A. Hall, $A n$ algebraic identity, Journal of the London Mathematical Society, vol. 11 (1936), p. 276.

${ }^{7}$ W. N. Bailey, Generalized Hypergeometric Series, Cambridge Tract, no. 32, p. 68.

${ }^{8}$ G. N. Watson, Dixon's theorem on generalized hypergeometric functions, Proceedings of the London Mathematical Society, (2), vol. 22 (1924), pp. xxxii-xxxiii (records for May 1923). See also W. N. Bailey, Generalized Hypergeometric Series, p. 13. 
gamma functions. However, a slight modification of this method does lead to a new and curious result, namely,

$$
\begin{aligned}
\sum_{n=0}^{\infty} \frac{\Omega\left(a q^{n}\right) \Omega\left(b q^{n}\right) \Omega\left(c q^{n}\right)}{\Omega\left(q^{n+1}\right) \Omega\left(a q^{n+1} / b\right) \Omega\left(a q^{n+1} / c\right)}(a q / b c)^{n} q^{n(n+1) / 2} \\
=\frac{\Omega(a) \Omega(b) \Omega(c)}{\Omega(a q) \Omega(a q / b c)}{ }_{3} \Phi_{2}\left[\begin{array}{c}
b, c,-q ; a q / b c \\
q a^{1 / 2},-q a^{1 / 2}
\end{array}\right] .
\end{aligned}
$$

The argument requires that a certain double series be absolutely convergent.

The left member of (2) is a modified basic series somewhat similar to some of the $q$-hypergeometric series studied by Jackson. ${ }^{9}$ Since Dixon's theorem sums a general well-poised ${ }_{3} F_{2}$ with unit argument, (2) cannot be considered a basic analogue of Dixon's theorem. This result is interesting inasmuch as it expresses what may be called a pseudo-basic series in terms of a function ${ }_{3} \Phi_{2}$. This seems to be the first result of its type to appear in the theory of basic series.

Agricultural and Mechanical College of Texas and UNIVERSITY OF NEBRASKA

${ }^{9} \mathrm{~F}$. H. Jackson, Summation of q-hypergeometric series, Messenger of Mathematics, vol. 50 (1921), p. 101. 